J. Lake Sci. (湖泊科学), $2006, \mathbf{1 8}(2): 134-138$

http:// www. jlakes. org. E-mail: jlakes@ niglas. ac.cn

(c) 2006 by Journal of Lake Sciences

\title{
人工湿地复合基质深度净水除磷实验"
}

\author{
李爱权 ${ }^{1,2}$, 李文朝 ${ }^{1}$, 潘继征 ${ }^{1}$, 熊 飞 ${ }^{1,2}$
}

( 1 : 中国科学院南京地理与湖泊研究所,南京 210008)

(2:中国科学院研究生院, 北京 100039)

\begin{abstract}
摘 要: 通过垂直上升流人工湿地模拟柱实验, 研究了泥炭、蛭石、砾石以不同体积比 (柱 1 比例 $1: 1: 3$, 柱 2 比例 $1: 1: 2$, 柱 3 比例 $1: 1: 1$, 柱 4 全为砾石)组成的复合基质对人工湿地出水的深度除磷效果. 结果表明,填充复合基质的柱 $1-3$ 对 总磷和正磷酸盐的处理效果较好, 去除率分别达到了 $77 \%$ 和 $90 \%$ 以上, 明显优于填充单一基质砾石的柱 4 (27\% 和 $21 \%)$; 蛭石、泥炭价格远高于砾石,从经济、有效的原则出发,用蛭石、泥炭、砾石以 $1: 1: 3$ 的体积比例混合作为复合填料 比较合理; 该复合基质适合于水质深度净化,在进水总磷浓度不超过 $0.1 \mathrm{mg} / \mathrm{L}$ 的情况下,除磷效果相当稳定, 可以保证 $0.02 \mathrm{mg} / \mathrm{L}$ 以下的出水浓度 (地表 III 类水质标准), 并达到 $80 \%$ 左右的去除率; 遇到高浓度磷污染负荷冲击时, 磷去除率 会降低; 该复合基质的除磷机制主要为吸附净化,单独使用易吸附饱和, 而与人工湿地中与湿地植物的吸收净化衔接起 来, 就能形成稳定持久的除磷效果.
\end{abstract}

关键词: 人工湿地;复合基质;除磷;深度净化

\section{Phosphorus removal efficiency of constructed wetland with different compound substrates treating pre-treated wastewater}

\author{
LI Aiquan ${ }^{1,2}$, LI Wenchao ${ }^{1}$, PAN Jizheng ${ }^{1} \&$ XIONG Fei ${ }^{1,2}$ \\ ( 1 :Nanjing Institute of Geography and Limnology, Chinese Academy of Sciences, Nanjing 21008, P. R. China) \\ (2: Graduate School, Chinese Academy of Sciences, Beijing 100039, P. R. China)
}

\begin{abstract}
In order to select suitable substrates to treat pre-treated wastewater by constructed wetland, the removal efficiencies of phosphorus of compound substrates which consisted of peat, vermiculite and gravel was investigated through the experiment of vertical up-flow simulation system. The composition of the substrates in four systems were $1: 1: 3,1: 1: 2,1: 1: 1$ and all gravel, respectively. The removal efficiency of $\mathrm{TP}$ and $\mathrm{PO}_{4}^{3-}-\mathrm{P}$ in system $1-3$ which were filled with compound substrates was up to $77 \%$ and $90 \%$, apparently better than system $4(27 \%$ and $21 \%)$. The substrates was suitable for profound purification. When the concentration of TP in inflow was lower than $0.1 \mathrm{mg} / \mathrm{L}$, the removal effect was very stable, it can make sure that the concentration of TP in outflow was lower than $0.02 \mathrm{mg} / \mathrm{L}$ and the removal efficiency was about $80 \%$. When it was struck by the inflow with high concentration phosphorus, the removal efficiency would decrease. The price of peat and vermiculite was much higher than gravel, so the composition of $1: 1: 3$ was a reasonable choice for economy and efficiency. The phosphorus re-

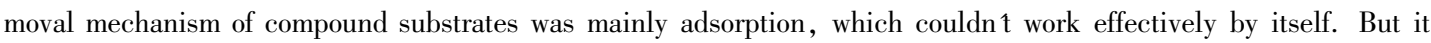
could form stable and sustainable phosphorus removal efficiency that having it work together with macrophyte in wetland.
\end{abstract}

Keywords : Constructed wetland; compound substrates; phosphorus removal; profound purification

人工湿地因其低投人、高效率及运行管理方便等优点越来越多地被用来处理多种类型的污水,如生活 污水、工业废水、农田污水和城市暴雨 ${ }^{[1-4]}$. 此外, 它们还可以在富营养化的地表水排人脆弱的自然水体之 
前削减其营养负荷 ${ }^{[5]}$. 利用人工湿地进行深度处理, 国内外都有不少相关报道, 但处理污水厂二级处理后 的出水并不多见. 本项实验研究主要围绕 “大理州洱源县城污水厂尾水深度净化湿地技术示范工程” 而开 展, 旨在为其土壤过滤系统研制人工基质配方,使其达到较好的除磷效果,出水达到国家地表水 III 类水质 标准. 从马料河和窑泥沟复合湿地的运行情况来看, 常规人工湿地不能使出水达到地表水 III 类水质, 其中 的主要限制因子为磷. 因此, 在此工程中增设了土壤渗滤系统. 土壤渗滤系统是湿地深度净化工艺中最重 要的环节, 其主要作用是去除残余的磷酸盐. 由于进人土壤渗滤系统的磷浓度已经相当低, 需要通过基质 对磷的吸附浓缩, 为水生植物提供营养, 并使出水达标. 对磷的吸附特性是笁选基质的首选条件, 有关研究 资料证明蛭石、泥炭对磷都有较好的吸附净化效果 ${ }^{[6]}$ (1). 本研究选用泥炭、蛭石、砾石为原料, 利用垂直上升 流人工湿地模拟柱, 研究它们在不同比例混合的情况下去除磷的效果,笁选最为经济、有效的基质配方.

\section{1 材料与方法}

\section{1 实验柱制作}

实验柱用内径 $90 \mathrm{~mm}$ 、高度 $1000 \mathrm{~mm}$ 的透明亚克力管制作. 管下端用亚克力封闭, 在其中心安装塑料水 咀, 作为进水口; 在距离顶部 $200 \mathrm{~mm}$ 的侧壁上安装塑料水咀, 作为出水口. 蛭石购于昆明花鸟市场, 泥炭 (由有机质、矿物质、水分组成, 其中有机质主要成分为腐质酸、纤维素、半纤维素、木质素) 取自滇池草海清 淤堆场, 砾石取自昆明市郊建材市场. 蛭石粒径 3-5 mm, 泥炭粉碎过篎 (60 目尼龙笁), 砾石粒径 5-8 mm (基质未进行除磷清洗). 在 4 个柱的底部填充 $100 \mathrm{~mm}$ 厚度鹅卵石 (粒径 $10-20 \mathrm{~mm}$ ), 我鸟卵石层上覆盖土 工布, 土工布上分别填充不同配比的复合基质厚度 $600 \mathrm{~mm}$. 在考虑砾石比较经济的前提下, 以砾石为主自 设三组不同比例的复合基质 (柱 1-3) 及一个参照组 (柱 4) (表 1 ).

\section{2 实验地点及水样}

实验于 2004 年 $10-12$ 月在中国科学院南京地 理与湖泊研究所抚仙湖工作站进行. 水样采用抚仙 湖马料河人工湿地出水, 其总磷含量为 $0.036-$ $0.246 \mathrm{mg} / \mathrm{L}$, 磷酸盐磷含量为 $0.025-0.242 \mathrm{mg} / \mathrm{L}$, 总氮为 $0.327-1.044 \mathrm{mg} / \mathrm{L}$, 氨氮为 $0.184-0.584$ $\mathrm{mg} / \mathrm{L}, \mathrm{COD}_{\mathrm{Cr}}$ 为 $7.69-17.38 \mathrm{mg} / \mathrm{L}, \mathrm{BOD}_{5}$ 为 $2.05-$ $3.80 \mathrm{mg} / \mathrm{L}, \mathrm{pH}$ 为 $7.57-8.20^{2}$.

表 1 复合基质配制的体积比例

Tab. 1 Composition of compound substrates

\begin{tabular}{cccc}
\hline 柱号 & 蛭石 & 泥炭 & 砾石 \\
\hline 柱 1 & $1 / 5$ & $1 / 5$ & $3 / 5$ \\
柱 2 & $1 / 4$ & $1 / 4$ & $2 / 4$ \\
柱 3 & $1 / 3$ & $1 / 3$ & $1 / 3$ \\
柱 4 & 0 & 0 & 1 \\
\hline
\end{tabular}

\section{3 实验运行管理}

原水经蠕动葲加压后, 从底部进水口进人模拟实验柱, 自顶部出水口溢流而出, 在填充基质层顶面覆盖 着 $100 \mathrm{~mm}$ 厚的自由水层. 采用恒定流量连续运行方式, 每个实验柱流量为 $7.56 \mathrm{ml} / \mathrm{min}$, 滞留时间 HRT 为 $3.8-4.2 \mathrm{~h}$. 从马料河人工湿地采集新鲜出水, 置于 $50 \mathrm{~L}$ 的塑料桶中作为实验供水, 每 $24 \mathrm{~h}$ 更换一次. 实验 连续运行 $30 \mathrm{~d}$, 每天采集一次水样进行两个平行样分析 (其中有几天未进行采样但系统仍然运行).

\section{4 水样采集与分析}

进水水样直接从供水塑料桶中采集, 用 $200 \mathrm{ml}$ 烧杯同时从每个实验柱出水口收集出水. 以上水样采集 后立即进行分析, 分析方法: 总磷 ( TP) ; 钼酸铵分光光度法 ( GB $11893-89)$; 磷酸盐磷 $\left(\mathrm{PO}_{4}^{3-}-\mathrm{P}\right)$; 磷钼蓝 分光光度法 ${ }^{[7]}$.

\section{2 实验结果}

连续 $30 \mathrm{~d}$ 的运行结果见表 2 . 原水总磷含量 $0.036-0.246 \mathrm{mg} / \mathrm{L}$, 平均为 $0.109 \mathrm{mg} / \mathrm{L}$, 其中 $75 \%$ 为磷酸

(1) 尹大强,袁东海. 构建人工湿地基质的净化氮磷特征研究. 中国水环境污染控制与生态修复技术高级研讨会论文 集 (2),2004:249-256.

(2) 数据由云南省玉溪市环境监测站提供. 
盐磷. 柱 $1-3$ 具有很好的除磷效果, 出水中总磷平均含量依次为 $0.022 \mathrm{mg} / \mathrm{L} 、 0.023 \mathrm{mg} / \mathrm{L} 、 0.025 \mathrm{mg} / \mathrm{L}$, 平 均去除率达到了 $79.3 \% 、 78.7 \% 、 77.2 \%$; 磷酸盐磷平均含量均为 $0.007 \mathrm{mg} / \mathrm{L}$, 平均去除率达到了 $91.4 \%$. 柱 4 除磷效果较差, 出水中总磷和磷酸盐磷平均含量分别为 $0.079 \mathrm{mg} / \mathrm{L} 、 0.065 \mathrm{mg} / \mathrm{L}$, 平均去除率分别为 $27.0 \% 、 20.9 \%$.

表 2 实验柱进出水中总磷及磷酸盐浓度 $(\mathrm{mg} / \mathrm{L})$

Tab. 2 Concentration of TP and $\mathrm{PO}_{4}^{3-}-\mathrm{P}$ in inflow and outflow through purification $\operatorname{system}(\mathrm{mg} / \mathrm{L})$

\begin{tabular}{|c|c|c|c|c|c|c|c|c|c|c|}
\hline \multirow{2}{*}{ 起始天数 } & \multicolumn{2}{|c|}{ 进水 } & \multicolumn{2}{|c|}{ 柱 1} & \multicolumn{2}{|c|}{ 柱 2} & \multicolumn{2}{|c|}{ 柱 3} & \multicolumn{2}{|c|}{ 柱 4} \\
\hline & $\mathrm{TP}$ & $\mathrm{PO}_{4}^{3-}-\mathrm{P}$ & $\mathrm{TP}$ & $\mathrm{PO}_{4}^{3-}-\mathrm{P}$ & $\mathrm{TP}$ & $\mathrm{PO}_{4}^{3-}-\mathrm{P}$ & $\mathrm{TP}$ & $\mathrm{PO}_{4}^{3-}-\mathrm{P}$ & $\mathrm{TP}$ & $\mathrm{PO}_{4}^{3-}-\mathrm{P}$ \\
\hline 1 & 0.246 & 0.242 & 0.089 & 0.029 & 0.092 & 0.030 & 0.116 & 0.035 & 0.228 & 0.209 \\
\hline 2 & 168 & 0.133 & 0.056 & 0.018 & 0.057 & 0.020 & 0.072 & 21 & 71 & 0.164 \\
\hline 3 & 0.148 & 0.123 & 0.048 & 0.014 & 0.050 & 0.015 & 0.056 & 0.017 & 0.142 & 0.121 \\
\hline 4 & 179 & 0.140 & 0.040 & 0.008 & 0.048 & 0.007 & 0.048 & 0.006 & 0.127 & 0.103 \\
\hline 5 & 0.176 & 0.139 & 0.033 & 0.008 & 0.036 & & 0.036 & 0.005 & 0.123 & 0.097 \\
\hline 6 & 0.127 & 0.101 & 0.031 & 0.007 & 0.031 & 0.007 & 0.029 & 0.006 & 0.110 & 0.100 \\
\hline 7 & & & & & & & 24 & 06 & 79 & 0.149 \\
\hline 8 & 0.100 & 0.066 & 0.020 & 0.005 & 0.019 & 0.004 & 0.017 & 0.004 & 0.092 & 0.052 \\
\hline 9 & 0.099 & 0.062 & 0.021 & 0.004 & 0.019 & 0.004 & 0.017 & 0.003 & 0.089 & 0.057 \\
\hline 10 & 80 & 0. & 16 & 44 & 12 & 03 & 12 & 04 & 62 & 054 \\
\hline 11 & 0.072 & 0.053 & 0.011 & 0.004 & 0.011 & 0.004 & 0.009 & 0.004 & 0.052 & 0.048 \\
\hline 12 & 0.048 & 0.033 & 0.008 & 0.004 & 0.009 & 0.003 & 0.010 & 0.004 & 0.033 & 0.031 \\
\hline 13 & 049 & 0.025 & 0.008 & 0. & 0.009 & 0. & 0.008 & 0.004 & 30 & 0.027 \\
\hline 16 & 0.115 & 0.085 & 0.008 & 0.003 & 0.012 & 0.002 & 0.011 & 0.002 & 0.021 & 0.019 \\
\hline 17 & 0.077 & & 0.009 & & & & & & 0.026 & 0.022 \\
\hline 19 & 0.195 & 0.156 & 0.012 & 0.005 & 0.018 & 0.008 & 0.015 & 0.008 & 0.066 & 0.037 \\
\hline 20 & 0.099 & 0.083 & 0.011 & 0.005 & 0.008 & 0.006 & 0.010 & 0.006 & 0.047 & 0.046 \\
\hline 22 & 0.036 & & & & & & & & & 0.031 \\
\hline 23 & 0.061 & 0.035 & 0.007 & 0.005 & 0.008 & 0.004 & 0.008 & 0.004 & 0.045 & 0.036 \\
\hline 27 & 0.041 & 0.029 & 0.009 & 0.002 & 0.013 & 0.003 & 0.013 & 0.003 & 0.037 & 0.026 \\
\hline 28 & 0.047 & 0.026 & 0.012 & 0.002 & 0.011 & 0.003 & 0.011 & 0.004 & 0.039 & 0.015 \\
\hline 29 & 0.038 & 0.027 & 0.010 & 0.004 & 0.009 & 0.003 & 0.010 & 0.004 & 0.026 & 0.018 \\
\hline 30 & 0.124 & & 0.021 & & 0.018 & 0.005 & 0.020 & 0.004 & 0.042 & 0.026 \\
\hline 均值 & 0.109 & 0.082 & 0.022 & 0.007 & 0.023 & 0.007 & 0.025 & 0.007 & 0.079 & 0.065 \\
\hline 除率 $(\%$ & - & - & 79.3 & 91.4 & 78.7 & 91.4 & 77.2 & 91.4 & 27.0 & 20.9 \\
\hline
\end{tabular}

\section{3 分析讨论}

\section{1 复合基质的合理配方}

从实验结果看,柱 $1-3$ 除磷效果均较好,平均出水浓度都在 $0.020 \mathrm{mg} / \mathrm{L}$ 以下,达到了地表 III 类水标准 (GB3838-2002); 对总磷的平均去除率分别为 79.3\%、78.7\% 和 $77.2 \%$, 远远高于一般人工湿地的总磷去 除率 $50 \%{ }^{[6]}$. 三个柱对磷酸盐的平均去除率差异甚微, 对总磷的平均去除率递减, 但减小幅度仅为 $2.6 \%$. 柱 4 除磷效果较差, 对总磷、磷酸盐的平均去除率仅为柱 3 的 $35.0 \%$ 和 $22.8 \%$. 因此, 用砾石作为单一填料 的构造湿地除磷效果较差, 按本研究的 3 种配比添加蛭石和泥炭可以提高除磷效率 2 倍左右. 根据以上实 验结果, 从经济、有效的原则出发, 用蛭石、泥炭、砾石以 $1: 1: 3$ 的体积比例混合作为复合填料比较合理. 能 否再降低蛭石和泥炭的含量并达到相近的净化效果,这有待进一步实验研究. 尹大强、袁东海1 1 对人工基质

(1) 尹大强, 袁东海. 构建人工湿地基质的净化氮磷特征研究. 中国水环境污染控制与生态修复技术高级研讨会论文 集(2),2004:249-256. 
吸附特性的研究表明, 蛭石的磷素饱和吸附量高达 $3711 \mathrm{mg} / \mathrm{kg}$, 泥炭也有较强的磷吸附能力 ${ }^{[6,9]}$, 因而本文 以砾石为基本填料、蛭石和泥炭为功能强化填料配置复合基质是科学合理的.

\section{2 复合基质对磷的富集特性}

在人工湿地中,复合基质的主要功能在于对低浓度营养盐的富集浓缩, 为湿地植物提供充足矿质营养. 蛭石 - 泥炭 - 砾石复合基质对磷的深度净化主要通过物理及化学吸附来实现, 实验初期磷去除率快速上升 (图 1), $7 \mathrm{~d}$ 以后平稳上升, $16 \mathrm{~d}$ 以后趋于平稳, $20 \mathrm{~d}$ 之后开始下降,可能是复合基质对磷的物理及化学吸附 已经开始出现饱和. 在人工湿地系统中, 基质富集、湿地植物的吸收与植物的收割是有机结合在一起的, 吸 附饱和的时间将被大大延长, 湿地磷饱和的时间通常大于十年 ${ }^{[10]}$.

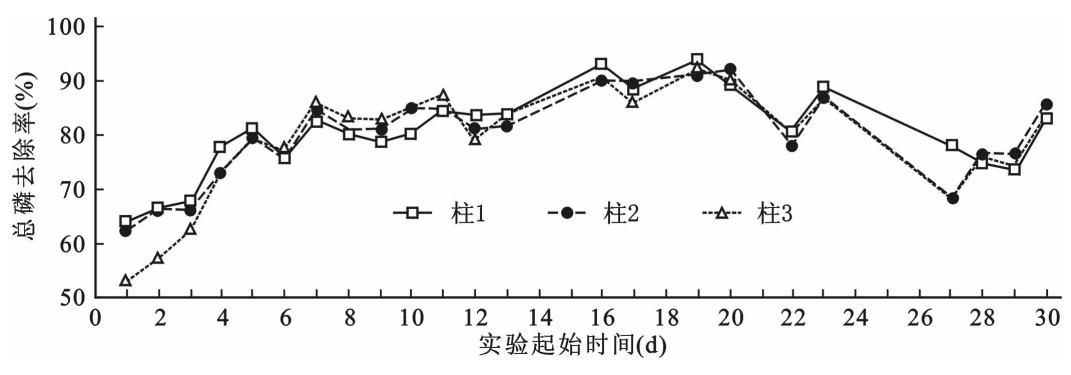

图 1 柱 $1-$ 柱 3 总磷去除率的变化过程

Fig. 1 Changing process of TP removal efficiency in system $1-3$

\section{3 复合基质除磷效果的稳定性}

实验开始后 5-20d 为复合基质正常发挥作用的阶段,将柱 $1-$ 柱 3 在这段时间内的进、出水总磷含量 进行回归分析, 结果见图 2. 显然, 进出水总磷浓度之间呈指数相关 $Y=0.0054 \mathrm{e}^{10.694 x}, R^{2}=0.7099$, 显著度 较高. 这说明, 复合基质对总磷的去除率并非恒定, 而是随着进水浓度的增高而降低. 当进水浓度不超过 $0.1 \mathrm{mg} / \mathrm{L}$ 时, 除磷效果相当稳定, 可以保证 $0.02 \mathrm{mg} / \mathrm{L}$ 以下的出水浓度, 并达到 $80 \%$ 左右的去除率; 当进水 浓度超过 $0.1 \mathrm{mg} / \mathrm{L}$ 时, 去除率波动较大( $52.8 \%-93.8 \%)$. 因此,这种复合基质适用于深度净化湿地工 程, 总磷的净化效果可以达到地表 III 类水质标准.

\section{4 复合基质在湿地系统除磷中的作用}

复合基质在湿地系统除磷中起中介的作用, 首 先复合基质从水体中通过物理化学吸附作用将磷 转移到基质中, 然后基质中的磷作为营养元素供植 物生长, 最后通过对植物的收割从湿地系统中去 除. 当然, 这些过程离不开微生物的协同作用.

由于本实验未引人湿地植物, 因此对磷的去除 是基质吸附和微生物同化两者共同作用的结果. 对 垂直流构建湿地的研究表明, 两条途径对磷去除的 贡献大小为: 基质 > 微生物, 砾石基质去除占 $20 \%$, 生物膜中微生物去除约占 $10 \%$. 基质对磷的吸 附分为: 物理吸附和化学吸附, 主要为化学机制 ${ }^{[6]}$.

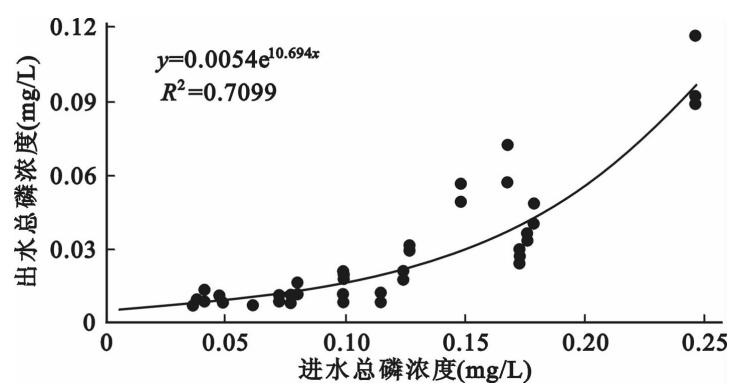

图 2 柱 $1-$ 柱 3 进、出水总磷浓度之间的函数关系 Fig. 2 Function relation between TP concentration in inflow and outflow in system $1-3$

蛭石去除废水中磷的作用包括了配位体交换吸附和 Ca-P 和 Mg-P 沉淀, 但以哪一种为主, 还有待于进一步 研究 ${ }^{[12]}$. 泥炭除了有发达的表面积对磷进行物理吸附外, 还可以发生交换反应去除磷 ${ }^{[11]}$. 研究表明, 泥炭 中含有一定量的金属离子 (如 $\mathrm{Fe}^{3+} 、 \mathrm{Ca}^{2+}$ 和 $\mathrm{Al}^{3+}$ ), 可通过形成溶解性的金属离子盐 $\mathrm{M}(\mathrm{H} 2 \mathrm{O})_{\mathrm{x}}(\mathrm{OH})_{\mathrm{y}} \mathrm{H}_{2}$ $\mathrm{PO}_{4}$ 及一系列不溶性磷酸钲等产生的固磷作用而大大提高其对磷的去除率 ${ }^{[13]}$. 微生物对磷的去除包括它 们对磷的正常同化 (将磷纳人其分子组成) 和对磷的过量积累. 微生物除磷主要是通过聚磷菌对磷的过量 
摄取作用而实现的 ${ }^{[14]}$.

\section{4 结论}

(1) 在砾石床人工湿地填料中添加蛭石和泥炭可以大幅度提高其除磷效率,将蛭石、泥炭、砾石按照 1 $: 1: 3$ 的体积比例混合配置的复合基质,在滞留时间为 4 小时左右时,其总磷平均去除率可以达到 $77.2 \%$, 磷酸盐平均去除率可以达到 $91.4 \%$.

（2）该复合基质适合于水质深度净化,在进水总磷浓度不超过 $0.1 \mathrm{mg} / \mathrm{L}$ 的情况下,除磷效果相当稳 定, 可以保证 $0.02 \mathrm{mg} / \mathrm{L}$ 以下的出水浓度（地表 III 类水质标准）, 并达到 $80 \%$ 左右的去除率. 遇到高浓度磷 污染负荷冲击时, 磷去除率会降低.

（3）该复合基质的除磷机制主要为吸附净化,单独使用易吸附饱和, 而与人工湿地中与湿地植物的吸 收净化衔接起来,就能形成稳定持久的除磷效果.

\section{5 参考文献:}

[1] Miklas Scholz, Jing Xu. Comparison of constructed reed beds with different filter media and macrophytes treating urban stream water contaminated with lead and copper. Ecological Engineering, 2002, 18: 385 -390 .

[2] Ji Guodong, Sun Tieheng, Zhou Qixing, et al. Constructed subsurface flow wetland for treating heavy oilproduced water of the Liaohe Oilfield in China. Ecological Engineering, 2002,18:459 - 465.

[3] Lee M Luckeydoo, Fausey N R, Brown L C, et al. Early development of vascular vegetation of constructed wetlands in northwest Ohio receiving agricultural waters. Agriculture, Ecosystems and Environment, 2002, $88: 89-94$.

[4] Carleton J N, Grizzard T J, Godrej A N, et al. Performance of a constructed wetlands in treating urban stormwater runoff. Water Environ Res, 2000 ,72(3) :295 - 304.

[5] Diederik P L Rousseau, Peter A Vanrolleghem, Niels De Pauw. Model-based design of horizontal subsurface flow constructed treatment wetlands: a review. Water Research, 2004, 38:1484-1493.

[6] 沈耀良. 泥炭及其在废水处理中的应用. 污染防治技术, 1997,10(1):36-39.

[7] 《水和废水监测分析方法》编委会. 水和废水监测分析方法 (第三版). 北京: 中国环境科学出版 社, 1989 .

[8] Verhoeven J T A, Meuleman A F M. Wetlands for wastewater treatment: Opportunities and limitations. Ecological Engineering, 1999, $12: 5-12$.

[9] 岳廷盛,张雪梅,王玉醌. 改性泥炭对磷吸附交换性能的研究. 铀矿冶,2000,19(4):276-279.

[10] Arthur F M Meuleman, Richard van Logtestijn, Gerard B J Rijs, et al. Water and mass budgets of a vertical-flow constructed wetland used for wastewater treatment. Ecological Engineering, 2003, 20 : 31 - 44.

[11] Lantzke L R, Mitchell D S, Heritage A D, et al. A model of factors controlling orthophosphate removal in planted vertical flow wetlands. Ecological Engineering, 1999, 12: 93 - 105.

[12] 邓雁希,许 虹, 黄 玲. 蛭石去除废水中磷酸盐的研究. 中国非金属矿工业导刊,2003,6:42-44.

[13] 崔理华,朱夕珍,骆世明等. 煤渣 - 草炭基质垂直流人工湿地系统对城市污水的净化效果. 应用生态 学报,2003,14(4):597-600.

[14] 吴晓否. 人工湿地废水处理机理. 环境科学,1995,16(3) :83-86. 